\title{
Energy loss of a high charge bunched electron beam in plasma: Simulations, scaling, and accelerating wakefields
}

\author{
J. B. Rosenzweig, N. Barov, ${ }^{*}$ M. C. Thompson, and R. B. Yoder \\ Department of Physics and Astronomy, UCLA, 405 Hilgard Avenue, Los Angeles, California 90095-1547, USA \\ (Received 13 March 2003; published 24 June 2004)
}

\begin{abstract}
The energy loss and gain of a beam in the nonlinear, "blowout" regime of the plasma wakefield accelerator, which features ultrahigh accelerating fields, linear transverse focusing forces, and nonlinear plasma motion, has been asserted, through previous observations in simulations, to scale linearly with beam charge. Additionally, from a recent analysis by Barov et al., it has been concluded that for an infinitesimally short beam, the energy loss is indeed predicted to scale linearly with beam charge for arbitrarily large beam charge. This scaling is predicted to hold despite the onset of a relativistic, nonlinear response by the plasma, when the number of beam particles occupying a cubic plasma skin depth exceeds that of plasma electrons within the same volume. This paper is intended to explore the deviations from linear energy loss using 2D particle-in-cell simulations that arise in the case of experimentally relevant finite length beams. The peak accelerating field in the plasma wave excited behind the finite-length beam is also examined, with the artifact of wave spiking adding to the apparent persistence of linear scaling of the peak field amplitude into the nonlinear regime. At large enough normalized charge, the linear scaling of both decelerating and accelerating fields collapses, with serious consequences for plasma wave excitation efficiency. Using the results of parametric particle-in-cell studies, the implications of these results for observing severe deviations from linear scaling in present and planned experiments are discussed.
\end{abstract}

DOI: 10.1103/PhysRevSTAB.7.061302

\section{INTRODUCTION}

The scheme of using electron beam-excited plasma waves, or plasma wakefields, to generate ultrahigh accelerating gradients for future linear accelerators, is known as the plasma wakefield accelerator (PWFA) [1]. In the past decade, work on the PWFA has concentrated on extending the PWFA from the linear regime [1,2], where the plasma oscillations can be considered small perturbations about an equilibrium, to the highly nonlinear "blowout" regime [3]. In the blowout regime, the plasma response to a beam that is much denser than the ambient plasma is violently nonlinear, as the plasma electrons are ejected from the path of the intense driving electron beam, resulting in an electron-rarefied region. This region contains only (nearly stationary) ions, and thus possesses linear electrostatic focusing fields that allow high quality propagation of both the driving [4-6] and accelerating beams. In addition, this region has superimposed upon it (TM) longitudinal electromagnetic fields, which, because the phase velocity of the wake wave is axisymmetric and nearly the speed of light, are independent of radial offset from the axis. Thus this wake may accelerate a trailing electron beam just as a traveling wave linac, with strong transverse focusing conveniently supplied by the plasma ions [3].

The beam dynamics in this scenario are termed linear, and are conceptually easy to understand using common

\footnotetext{
${ }^{*}$ Current address: Department of Physics, Northern Illinois University,

DeKalb, Illinois 60115, USA.
}

PACS numbers: 52.40.Mj, 52.75.Di, 29.17.+w, 29.27.-a

tools. On the other hand, until recently the plasma dynamics of the PWFA blowout regime, with their extreme nonlinearity, have been only qualitatively understood, mainly through both fluid and particle-in-cell (PIC) simulations $[3,7,8]$. The companion work [2] to this paper has made progress in moving toward an analytical understanding of the plasma response to very large beam charges.

Despite the lack of analytical models for the nonlinear plasma response, it has been noted in a variety of studies that the accelerating and decelerating fields associated with the blowout regime obey a Cerenkov-like scaling [7,9-11]. This scaling [12] predicts that the fields which produce energy loss and gain in the system are proportional to the square of the characteristic maximum frequency in the system (the plasma frequency, $\omega_{p}^{2}$ ). The efficient excitation of an oscillatory system by a pulse occurs when the pulse is short compared with the oscillator period, requiring the rms pulse length $\sigma_{z}$ to obey the constraint $k_{p} \sigma_{z} \leq 2$. Thus linear scaling implies that the PWFA decelerating and (assumed proportional) acceleration fields should be related to the pulse length by $E_{z} \propto \sigma_{z}^{-2}$.

This prediction provides motivation for recent experiments that employ bunch compressors to reduce $\sigma_{z}$. In recent measurements with compressed beam at FNAL [13], the trailing portion of a $5 \mathrm{nC}, 14 \mathrm{MeV}, \sigma_{z}=$ $1.2 \mathrm{~mm}$ beam pulse was nearly stopped in $8 \mathrm{~cm}$ of $n_{0} \cong 10^{14} \mathrm{~cm}^{-3}$ plasma, a deceleration rate of over $150 \mathrm{MeV} / \mathrm{m}$, obtained in the PWFA blowout regime. Despite the nonlinearity of the plasma motion in this 
experiment, as well as other recent measurements at SLAC, the linear scaling of wakefields with respect to charge seems to have been well upheld [14].

On the other hand, in the future, beams compressed to ever-shorter lengths will be employed in experimental scenarios. For example, in the context of the SLAC E164 experiment [15] it has been proposed to use a beam that is compressed to as short as $12 \mu \mathrm{m}$ in $\mathrm{rms}$ length, resulting in over $10 \mathrm{GeV} / \mathrm{m}$ acceleration gradients. This experimental test is quite important, as it is a milestone on the road to the so-called "after-burner" concept [16], in which one may use a very high energy drive beam to double the energy of a trailing beam population, e.g., converting the Stanford Linear Collider at SLAC from $50 \mathrm{GeV}$ per beam to $100 \mathrm{GeV}$. The use of such short drive beams, and verification of scaling, could thus help realize such an ambitious goal.

The present work is concerned with two aspects of the problem of plasma wakefield acceleration and its scaling to ultrahigh fields. The first is to show that the assertion of a linear relationship between the exciting beam charge and the amplitude of the excited wakefields previously deduced from simulations does not hold when the charge is high enough. The second is to explore the implications of the approach to understanding the physics of the PWFA in the very nonlinear regime suggested by recent analytical work by Barov et al. [2] that examines a limit of this regime, that of an infinitesimally short ( $\mathrm{rms}$ bunch length $\sigma_{z} \rightarrow 0$ ) driving beam. This exploration is accomplished through particle-in-cell simulations that serve to subject the analytical results to verification wherever possible, and also to extend these results beyond the applicability of the analysis to the experimentally relevant realm of finite bunch lengths.

This analytical work in Ref. [2] is concerned with only the energy loss of an infinitesimally short beam. In the course of this analysis, a normalized charge was defined which delineates, as it approaches and then exceeds unity, when one should expect nonlinear effects. These nonlinear effects notably include plasma electron relativistic effects, magnetic forces on plasma electrons, and large plasma electron density modulations.

The normalized beam charge may be defined as follows:

$$
\tilde{Q}=4 \pi k_{p} r_{e} N_{b}=\frac{N_{b} k_{p}^{3}}{n_{0}}
$$

Equation (1) indicates that $\tilde{Q}$ is the ratio of the beam charge to the plasma electron charge located within a volume of a cubic plasma skin depth, $k_{p}^{-3}$. Here, the plasma skin depth is defined as $k_{p}=c / \omega_{p}$, where the electron plasma frequency is $\omega_{p}=\sqrt{4 \pi e^{2} n_{0} / m_{e}}$, and $n_{0}$ is the ambient plasma electron density.

Linear theory indicates that in the PWFA the plasma electron perturbations excited by the beam which give rise to the longitudinal electric field on the beam are contained within a region of approximate length and radius $k_{p}^{-1}$. Thus the relevant total number of plasma electrons that respond to the beam is limited to approximately $n_{0} k_{p}^{-3}$, and the comparison to the beam population $N_{b}$ is natural. When the ratio $\tilde{Q}$ exceeds unity, it is clear that the plasma field response to the beam, even with complete rarefaction of the beam channel (a region smaller than $k_{p}^{-2}$ ) is limited to a level lower than the beam fields themselves. When $\tilde{Q} \gg 1$, the beam fields are therefore dominant. To make a comparison to the analysis of Ref. [2], we adopt here the normalized notation employed in that work. All time intervals in the analysis are normalized to $\omega_{p}^{-1}$ and spatial distances to $k_{p}^{-1}$, all densities to $n_{0}$, velocities to $c$, momenta to $m_{e} c$ all current densities to $e n_{0} c$, and all fields to $m_{e} c \omega_{p} / e$. All normalized variables are indicated by the tilde symbol, e.g., $\tilde{H}_{\phi} \equiv e H_{\phi} / m_{e} c \omega_{p}$ and $\tilde{Q}$. In this regard, it should be noted that if $\tilde{Q} \ll 1$, the plasma response should be linear, and all other normalized variables are small compared to unity. Nonlinear features appear in the response when $\tilde{Q}$ approaches or exceeds 1 .

Some of these nonlinear features can be anticipated easily; as noted before, the plasma electrons become rarefied from the beam channel. We can comment in this regard that it is assumed, for the moment, that the beam is both radially narrow $k_{p} \sigma_{r} \ll 1$ and short $k_{p} \sigma_{z}<2$, and thus if $\tilde{Q}$ exceeds unity then the ratio of the beam-to-plasma density, $n_{b} / n_{0}=\tilde{Q} /(2 \pi)^{3 / 2} k_{p} \sigma_{z}\left(k_{p} \sigma_{r}\right)^{2}$, is greater than 1 . Under these assumptions, which are most often, but not always, obeyed in experimental conditions, the statement that $\tilde{Q}$ is much greater than unity implies blowout regime conditions, where the beam is much denser than the plasma. With such conditions, the plasma electrons are then ejected from the beam channel. For recent experiments in the blowout regime $[13,14]$, the value of $\tilde{Q}$ is in the range of 1.5-4; for ongoing experiments, $\tilde{Q}$ may increase by over an order of magnitude.

It may also be anticipated that accompanying this large amplitude plasma density modulation implies a large velocity response in the plasma electrons themselves. Indeed, it has been observed in simulations that the plasma electrons attain relativistic velocities as they are ejected from the beam channel $[3,8]$. What has not been appreciated before in the literature is that a strong component of the imparted relativistic momentum impulse is predicted to be in the forward longitudinal $(+z)$ direction. For infinitesimally short (longitudinal $\delta$-function) beams, the longitudinal momentum impulse is asserted to be [2] increasingly dominant; the radial and longitudinal components are related by

$$
\Delta \tilde{p}_{z}=\frac{1}{2} \Delta \tilde{p}_{r}^{2}
$$

where $\tilde{p}=p / m_{e} c$ is the normalized momentum. In contrast, in the case of excitation of small amplitude plasma 
waves, the initial longitudinal motion of the plasma electrons due to the introduction of the beam pulse is always in the negative direction $(-z)$.

The analysis presented in Ref. [2] further asserts that the fields directly behind a $\delta$-function beam [located at the zero of the normalized variable $\left.\xi=k_{p}\left(z-v_{b} t\right)\right]$ are identical to those obtained from linear theory. In the case analyzed in Ref. [2], this infinitesimally short beam has uniform surface charge density up to a normalized radius $\tilde{a}=k_{p} a$. The longitudinal electric field is obtained through a process that initially requires calculation of the magnetic field integral over the beam pulse, $\mathbf{H}=$ $\int_{-\varepsilon}^{\varepsilon} \tilde{H}_{\phi} d \xi$. The functional form of $\mathbf{H}$ is of modified Bessel function type [2]. Further, $\mathbf{H}$ is asserted to be, in the $\delta$-function beam limit, the radial momentum impulse imparted to the plasma electron, $\Delta \tilde{p}_{r}=\mathbf{H}$.

The analysis also predicts that the longitudinal momentum impulse imparted to the beam is $\Delta \tilde{p}_{z}=\frac{1}{2} \mathbf{H}^{2}$. Thus, the normalized velocity components associated with these momenta are $\tilde{\boldsymbol{v}}_{r}=\mathbf{H} /\left(1+\frac{1}{2} \mathbf{H}^{2}\right)$ and $\tilde{\boldsymbol{v}}_{z}=$ $\frac{1}{2} \mathbf{H}^{2} /\left(1+\frac{1}{2} \mathbf{H}^{2}\right)$. The equation of continuity for the plasma electrons in turn implies an enhancement of the plasma electron density immediately being the driving beam, $\tilde{n}=\left(1-\tilde{v}_{z}\right)^{-1}=1+\frac{1}{2} \mathbf{H}^{2}$. The combined effects of the crossed electric and magnetic fields causes a "snowplowing" of the plasma electrons, resulting in enhanced density. Thus the normalized transverse current density is given by $\tilde{J}_{r}=\tilde{n} \tilde{v}_{r}=\mathbf{H}$, which is identical to that obtained in a linear (nonrelativistic) analysis. This linearlike scaling is caused by the fortuitous cancellation of two effects: the radial velocity is limited by relativistic effects, while the density grows via the snowplow effect, exactly compensating for the reduced radial velocity response. These effects were explored by some preliminary simulations in Ref. [2], which unequivocally showed initial forward longitudinal motion in the plasma electrons, as well as concomitant density increase directly behind the beam.

Of primary importance, however, is the energy loss of the beam, and thus the longitudinal electric field at the beam, in the plasma. In the case of the $\delta$-function beam, the prediction for the field directly behind the disklike beam [2], in the limit $\tilde{a} \ll 1$, is

$$
\begin{aligned}
\left.\tilde{E}_{z}(\tilde{r})\right|_{\xi=\varepsilon^{-}} & =\int_{\infty}^{\tilde{r}} \mathbf{H}\left(\tilde{r}^{\prime}\right) d \tilde{r}^{\prime}=\frac{\tilde{Q}}{\pi \tilde{a}^{2}}\left[1-\tilde{a} K_{1}(\tilde{a}) I_{0}(\tilde{r})\right], \quad \tilde{r}<\tilde{a}, \\
& \cong \frac{\tilde{Q}}{\pi \tilde{a}^{2}}\left[1-\tilde{a} K_{1}(\tilde{a})\right] \cong \frac{\tilde{Q}}{2 \pi} \ln \left(\frac{1.123}{\tilde{a}}\right) .
\end{aligned}
$$

The energy loss gradient associated with this field is given, for a $\delta$-function beam, by one-half of the longitudinal force directly behind the beam $[2,17], \tilde{F}=\tilde{E}_{z} / 2$. It is predicted to be linear in charge regardless of the size of $\tilde{Q}$.

This scaling in energy loss for the limiting case of the $\delta$-function beam has been suggested as an illuminating

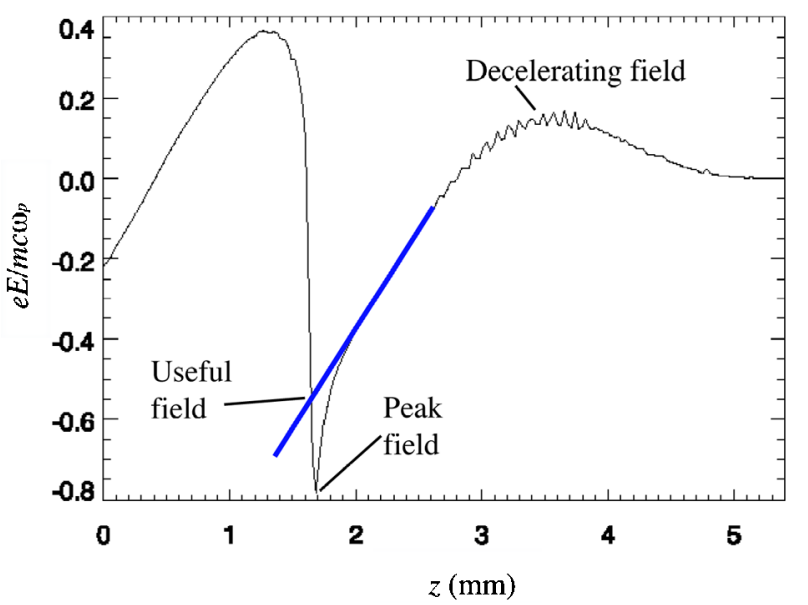

FIG. 1. (Color) The longitudinal field profile given by PIC simulation for PWFA excitation in the blowout regime. The decelerating field, peak accelerating field, and a defined "useful field," which avoids the narrow spike region, are indicated in the drawing.

model that helps explain the persistence of linearlike scaling of both the deceleration of a drive beam in the blowout regime of the PWFA and in the subsequent available acceleration fields. In order to test these claims, in this paper we quantitatively explore, with PIC simulations using the 2D axisymmetric codes OOPIC [8] and MAGIC [18], the dependence of these longitudinal fields on the normalized charge $\tilde{Q}$.

In order to perform such an analysis, several definitions associated with the simulations must be introduced. The typical on-axis longitudinal field profile excited in the blowout regime is shown in Fig. 1. Three measures of the field amplitudes are given in this figure: the well-behaved decelerating field inside of the driving beam, ${ }^{2}$ the peak accelerating field, which is characterized by a narrow spike, and "useful" field, that which directly precedes the spike. The decelerating field is investigated in detail in this paper to make a strong connection to the analysis in Ref. [2]. The second measure of acceleration given is termed useful because the acceleration associated with the spike is an extremely narrow region, with negligible stored energy, and therefore of very limiting use for efficiently accelerating a real beam. It will be seen that the scaling of plasma wakefield amplitudes, as measured in particular by the peak accelerating field spike, follows linearlike behavior well into the nonlinear regime. The mechanisms behind this anomalous scaling are explored, as are the ways in which they fail in the extremely nonlinear limit.

\footnotetext{
${ }^{2}$ One must take care that the PIC simulation parameters (mesh size and simulation particle number) are chosen to give well-behaved decelerating fields. Simulation noise problems become more serious when the beam charge is raised to larger than unity.
} 
Before examining the scaling of the longitudinal fields in the nonlinear regime of the PWFA, we begin our discussion of simulation results by looking at the qualitative aspects of the plasma electron response. In this way, we can critically examine aspects of the predictions of the nonlinear theory given in Ref. [2] using a method that is independent of the fluid analysis employed therein.

\section{ASPECTS OF THE PLASMA RESPONSE}

In order to explore the predictions concerning the nonlinear plasma response from the analytical $k_{p} \sigma_{z} \ll 1$ result, we have performed a series of simulations using the fully relativistic PIC codes MAGIC and OOPIC. Two codes were used initially to check consistency; both codes gave essentially the same answers for all comparisons. Both codes were run with $15 \mathrm{GeV}$ initial beam energy, to guarantee that the beam is ultrarelativistic and to suppress transverse evolution of the beam distribution. The first investigation undertaken using MAGIC concerned the validity of the physical model we have deduced from the analysis of Ref [2]. In particular, as one never expects the snowplow effect from linear theory, it is important this is observed. The two main characteristics of snowplow are (1) a forward velocity component and (2) a plasma electron density increase, both occurring in the region directly behind the beam. These effects are noted in beams of moderate length $\left(k_{p} \sigma_{z} \sim 1\right)$.

In order to observe this effect most strongly, we next display the result of a short (to approach the $\delta$-function limit) beam simulation. In Fig. 2, we display a simulation with high charge, $\tilde{Q} \cong 20$, ultrashort, $k_{p} \sigma_{z}=0.11$, beam, which is also narrow, $k_{p} a=0.2$. Note that the

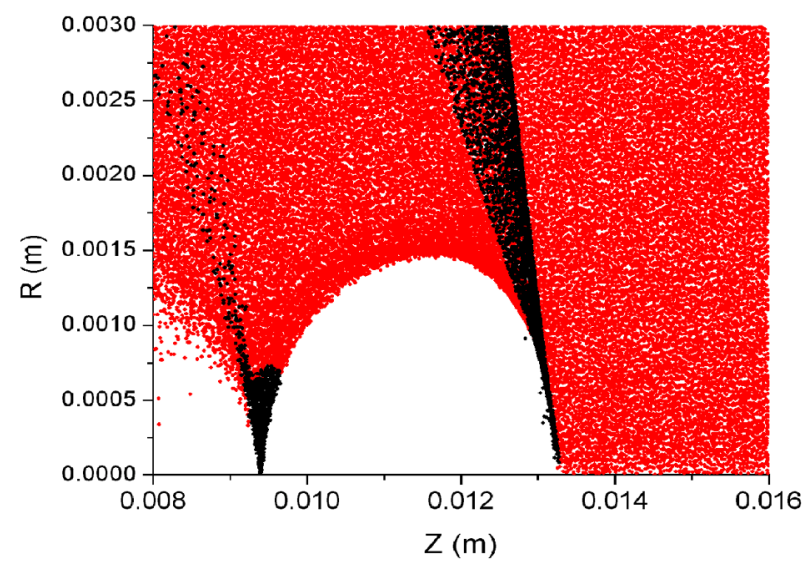

FIG. 2. (Color) Configuration space from MAGIC cylindrically symmetric PIC simulation with $\tilde{Q} \cong 20, k_{p} \sigma_{z}=0.11, k_{p} a=$ 0.2 , and beam center at $z=1.33 \mathrm{~cm}$. Color code indicates electron positions that have relativistic positive momenta $p_{z} / m_{e} c>1$ in black, with all other plasma electrons colored red. The initially accelerated plasma electrons are just ahead of the blowout region, where radial motion moves the electrons away from the beam channel. "shock front" shown in this case, which consists of electrons moving both forward and radially outward at relativistic speeds (the selected electrons must have $\tilde{p}_{z}>1$ ) is not a representation of the initial disturbance, which is localized around the longitudinal position of the beam. The front is canted because many of the electrons in it, which are located far from the axis, originated quite close to the axis. In fact, one may expect that the leading edge of this front consists of particles that are ejected with relativistic transverse velocity, and thus the edge must have roughly a $45^{\circ}$ angle with respect to the axis (recall that the electrons are launched by a source moving at nearly light speed). This angle may be verified from inspection of Fig. 2; note the difference in radial and longitudinal scales. The ultimate trajectory of these ejected electrons impacts the possible acceleration available in the wakefield behind the beam. We will return to this subject below.

In order to more quantitatively explore the predictions of the analysis, as well as the preliminary simulation work-concerning only the region near the beam itself-given in Ref. [2], a series of additional simulations was undertaken with OOPIC. These calculations employed had the following cylindrically uniform beam shape: flattop radial distribution of width $k_{p} a=0.2$ and flattop longitudinal of length $k_{p} l_{z}=0.1$ (effectively much shorter than even in the example of Fig. 2). This type of beam, which is as close as possible obtainable (given the constraints of computational stability) to the ideal $\delta$ function, was then scaled in charge $\tilde{Q}$ upwards from 0.2 , to 2,20 , and 200 .

To illustrate the relevant physical processes, we begin by plotting the plasma electron density as well as the plasma longitudinal current density, for the $\tilde{Q}=200$ case, in Fig. 3. It can be seen from Fig. 3(a) that the plasma density is strongly snowplowed in the vicinity of the driving beam, with a strong component of the longitudinal current density located there. Within this high forward-current region, the plasma density [Fig. 3(b)] is roughly 6 times the ambient density, indicating the severity of the snowplow in this highly nonlinear case.

Both the plasma electron density and the longitudinal currents show a strong localization around the rarefied region in Fig. 3. It can be seen that the plasma disturbance is surprisingly well behaved even during such large amplitude motion. The nonlinear plasma motion coheres fairly well until the strong wave-breaking event located where the plasma electrons return to the axis. Note that portions of the disturbance propagate to large radial offset; these artifacts are associated with the electrons that are strongly ejected by the beam, and also with other regions having large longitudinal current density (both positive and negative).

In order to examine the details of the immediate plasma response to the beam, we plot the current densities directly behind the driving beam that resulted 


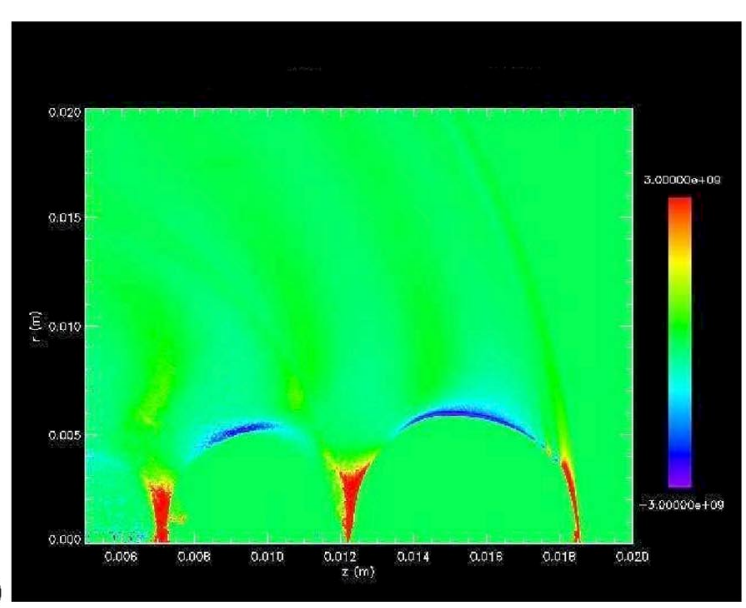

(a)

(b)

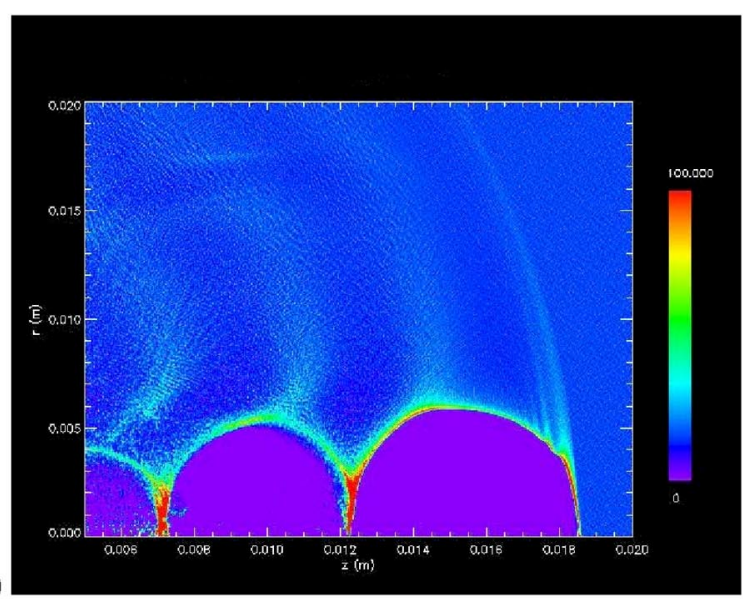

FIG. 3. (Color) OOPIC simulation of the $\tilde{Q}=200$ case, drive beam having uniform distribution of width $\tilde{a}=0.2$ and length $\tilde{l}_{z}=0.1$. False color (a) plasma longitudinal current density (color map key on right indicates current density in $\mathrm{A} / \mathrm{m}^{2}$ ) and (b) plasma electron density (color map key on right, ambient level is at 15, indicated in blue).

from these simulations. This macroscopic quantity reflects both the density and velocity state of the plasma electrons, and in the analytical case $\tilde{J}_{r}$ also represented the magnetic field response.

It can be seen in Fig. 4 that the $\tilde{Q}=0.2$ case shows a small amount of longitudinal current density directly behind the beam, even though the beam-plasma system may be naively thought to be in the linear response regime. Inspection of our expressions for the velocity and current density indicates that this is not a completely linear system; the peak velocity induced at beam edge is expected to be $0.15 c$ even in this case. The nonlinearity arises even with a relatively small charge, because the peak beam density is 1.6 times $n_{0}$.

In the higher $\tilde{Q}$ cases, however, nonlinear effects are even more pronounced. In Fig. 5 we plot, along with $\tilde{J}_{z}$ and $\tilde{J}_{r}$, the quantity which relates the two in the $\delta$-function beam limit, $\tilde{J}_{z}=\frac{1}{2} \tilde{J}_{r}^{2}$; the comparison is quite good. Note that in Fig. 4 the longitudinal current density

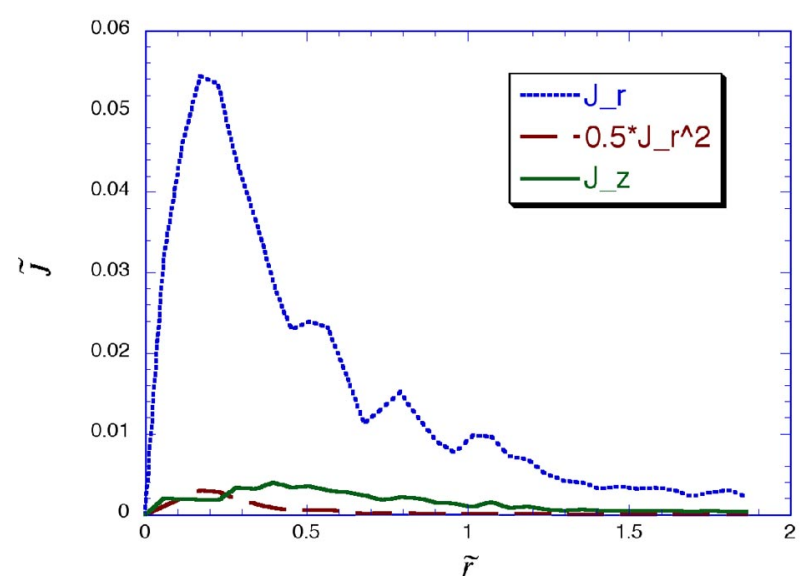

FIG. 4. (Color) Current densities just behind driving beam with $\tilde{Q}=0.2$, uniform distribution of width $\tilde{a}=0.2$, and length $\tilde{l}_{z}=0.1$. Analytical prediction $\tilde{J}_{z}=\frac{1}{2} \tilde{J}_{r}^{2}$ from theory also shown.

$\tilde{J}_{z}$ was small enough that its amplitude was dominated by noise, and the comparison is not as good.

Finally, we show the current densities associated the quite nonlinear $\tilde{Q}=20$ case in Fig. 6 . Note that the current densities have moved away from the beam distribution $(\tilde{r}>\tilde{a}=0.2)$ significantly during the beam passage. This further indicates that the induced decelerating electric field should be smaller, as the radial currents associated with the induced $\tilde{E}$ are reduced by the diminishing of the coupling (by simple proximity arguments) to the driving electron beam charge. It is also striking to note that normalized current densities $\tilde{J}$ are well larger than unity-without the snowplow enhancement of the plasma electron density, this is strictly forbidden. For the $\tilde{Q}=200$ case, $\tilde{J}_{z}$ is, as expected, even larger (over 11 ), and exceeds $\tilde{J}_{r}$ by a factor of 2 . Thus, as predicted by analytical results [given here in Eq. (2)], the longitudinal

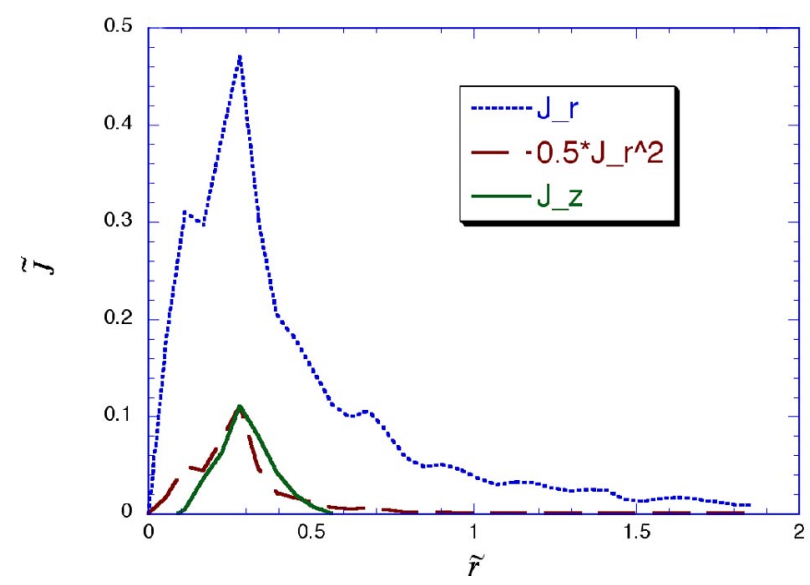

FIG. 5. (Color) Current densities just behind driving beam with $\tilde{Q}=0.2$, uniform beam of width $\tilde{a}=0.2$, length $\tilde{l}_{z}=0.1$. Analytical prediction $\tilde{J}_{z}=\frac{1}{2} \tilde{J}_{r}^{2}$ also shown. 


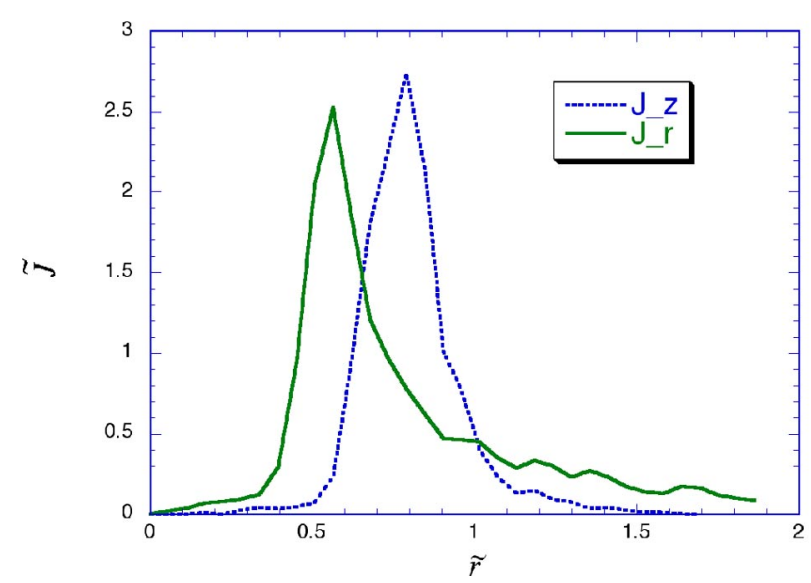

FIG. 6. (Color) Current densities just behind driving beam with $\tilde{Q}=20$, uniform distribution of width $\tilde{a}=0.2$, and length $\tilde{l}_{z}=$ 0.1 . Note that the currents have moved away from the beam distribution $(\tilde{r}>\tilde{a}=0.2)$ significantly during the beam passage.

current density eventually exceeds the radial current density when $\tilde{Q}$ becomes very large.

\section{DECELERATION AND ACCELERATION SCALING STUDIES: ULTRASHORT BEAM}

In order to evaluate the characteristics of the induced electric field driven by an ultrashort beam, we summarize the results of the parametric scan in $\tilde{Q}$ (from 0.02 to 200) in Fig. 7. This scan is an example of ideal scaling, in which the beam geometry and plasma density are held constant, while the charge is increased. In this figure, we plot the average on-axis deceleration experienced by the driving beam, and the associated prediction of linear theory, which in the short beam limit is given by $\tilde{F}_{\text {dec }} \cong$ $\left(\tilde{Q} / 2 \pi \tilde{a}^{2}\right)\left[1-\tilde{a} K_{1}(\tilde{a})\right]$. We choose the average deceleration as a relevant measure to accurately quantify the energy imparted to the plasma by the beam passage. This choice also allows us to connect the simulations with both the analytical $\delta$-function beam limit and also the case of longer, Gaussian beams discussed below.

In addition, we also plot three measures of the accelerating field for each case: two from the simulation, the peak acceleration, and the useful acceleration; also, the peak acceleration from the predictions of linear theory, obtained (as was also done for the average deceleration) by performing a convolution integration [17] over the drive beam, using the result of Eq. (3) as the Green function, $\tilde{F}_{\max } \cong\left(\tilde{Q} / \pi \tilde{a}^{2}\right)\left[1-\tilde{a} K_{1}(\tilde{a})\right] \cong 2 \tilde{F}_{\mathrm{dec}}$, in the integral. This procedure is of course not valid for the nonlinear response, but is employed only to extrapolate the predictions of linear theory.

Figure 7 shows some expected and some unexpected behavior. First, we note with satisfaction that until $\tilde{Q}>20$, the average deceleration observed in simulation is very close to that predicted by linear theory. This is a direct verification of the extrapolation of the linear re-

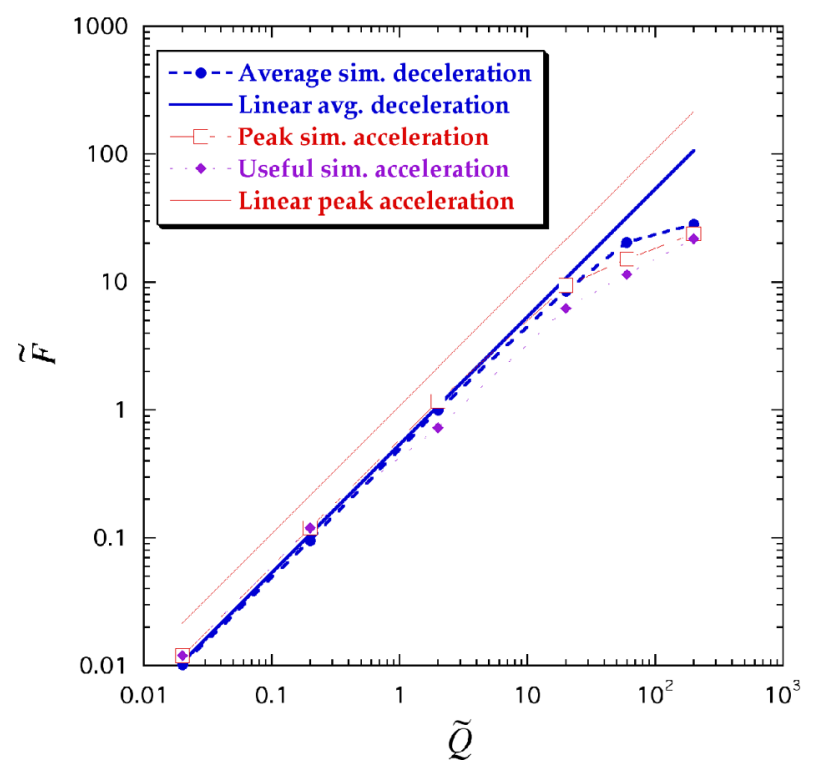

FIG. 7. (Color) The average normalized energy loss rate of $\tilde{F}_{\mathrm{dec}}=e\left|\left\langle E_{z}\right\rangle\right| / m_{e} c \omega_{p}$ of an electron beam with $\tilde{l}_{z}=0.1, \tilde{a}=$ 0.2 , as a function of $\mathscr{Q}$, from linear theory (solid bold line) and self-consistent PIC simulation (circles), the peak accelerating field behind the beam, $\tilde{F}_{\max }=e\left|E_{z \text {,max }}\right| / m_{e} c \omega_{p}$, from linear theory (solid fine line) and PIC simulation (squares), and the useful field for acceleration (diamonds), defined by the geometry in Fig. 1.

sponse of the decelerating field predicted by the analysis of the $\delta$-function beam. Above $\tilde{Q}=20$, the decelerating field is smaller than one expects from this limiting case, because (as already noted in the current response shown in Fig. 5) the plasma electrons may already be rarefying the near-beam region during the passage of the beam. This effect is not possible within the analytical modelthe electrons do not notably change position within the time of the beam passage.

The peak, as well as the useful, accelerating field observed in the simulations is, according to Fig. 7, well below (nearly a factor of 2) that expected from linear theory, even for small $\tilde{Q}$. This effect is due to nonlinear response having much to do with the very high density $\left(n_{b}=1.6 n_{0}\right.$ even for $\left.\tilde{Q}=0.02\right)$ in such a short beam. Aspects of the microscopic mechanisms for diminishing of the acceleration field are evident in Fig. 3, and are discussed in conjunction with the results shown in Fig. 8. This type of nonlinearity is not observed for small $\tilde{Q}$ cases in the longer beam simulations discussed in the following sections - such cases agree quite well with linear theory. We now turn to our examination of these simulation results.

\section{IDEAL SCALING WITH A GAUSSIAN BEAM-PLASMA SYSTEM}

While the $\delta$-function beam limit is relevant to attempting a validation of the theoretical analysis, it is not of 


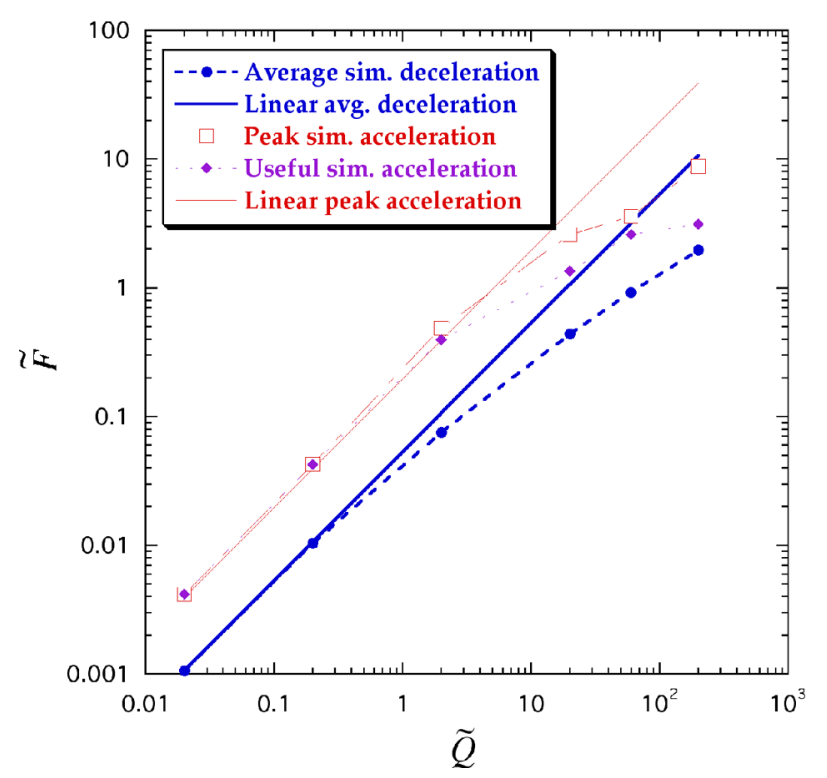

FIG. 8. (Color) The average normalized energy loss rate of $\tilde{F}_{\text {dec }}=e\left|\left\langle E_{z}\right\rangle\right| / m_{e} c \omega_{p}$ of a Gaussian-current electron beam with $k_{p} \sigma_{z}=1.1, \tilde{a}=0.2$, as a function of $\tilde{Q}$, from linear theory (solid bold line) and self-consistent PIC simulation (circles), the peak accelerating field behind the beam, $\tilde{F}_{\max }=$ $e\left|E_{z, \max }\right| / m_{e} c \omega_{p}$, from linear theory (solid fine line) and PIC simulation (squares), and the useful field for acceleration (diamonds).

highest practical interest in bunched beams, which generally have a Gaussian-current distribution, $\rho_{b}(z) \propto$ $\exp \left(-z^{2} / 2 \sigma_{z}^{2}\right)$. Further, it has often been argued that one should choose the plasma density such that $k_{p} \sigma_{z} \cong$ 1 to optimize drive beam energy loss and accelerating beam energy gain in a PWFA [1,3-5,9-11]. In order to explore the deviation in plasma response from the analytical $\left(k_{p} \sigma_{z} \rightarrow 0\right)$ result, therefore, we have performed a series of OOPIC simulations. We again take the beam of radius $\tilde{a}=0.2$ (again keeping the transverse beam profile uniform, to compare with the extrapolations of linear theory), and Gaussian-current profile with $k_{p} \sigma_{z}=1.1$. We have scanned the charge from $\tilde{Q}=0.02$ to 200 , values indicating linear to very nonlinear cases.

In Fig. 8, the average on-axis decelerating field, as calculated from the simulation through $\left.\left(2 \pi \sigma_{z}\right)^{-1} \int e E_{z}(z)\right|_{r=0} \exp \left(-z^{2} / 2 \sigma_{z}^{2}\right) d z$, again compares well with the linear theory prediction of $\left(\tilde{Q} / 2 \pi \tilde{a}^{2}\right)[1-$ $\left.\tilde{a} K_{1}(\tilde{a})\right] \exp \left(-k_{p}^{2} \sigma_{z}^{2}\right)$, until $\tilde{Q}$ exceeds 2 . It should be noted that $\tilde{Q}=2$ is just into the blowout regime, as the beam is 2.3 times denser than the plasma. One in fact would expect that the linear prediction would begin to fail for $\tilde{Q}$ an order of magnitude smaller, but it does not, because of the snowplow effect. The enhancement of the coupling due to snowplowing of the plasma electrons is a reflection of the longer interaction time, since the plasma electrons are traveling longitudinally at relativistic speed, and thus stay in contact with the beam longer. Thus, even though the plasma electrons may move radially outward away from the beam, the beam-plasma coupling stays anomalously strong for moderately large $\tilde{Q}$ (less than 10). At very large $\tilde{Q}$, this coupling, as measured by the deceleration, diminishes notably - it is an order of magnitude smaller than predicted by extrapolated linear theory.

Examination of the acceleration amplitudes in the simulation shows several interesting features. The first is that if one relies on the peak as a measure of the acceleration, the spike that occurs at the back of the accelerating region misleadingly indicates linearlike response until $\tilde{Q}=20$. In fact, the spike in the peak field that we have discussed above is magnified in the more nonlinear cases, causing a field enhancement relative to linear theory for $\tilde{Q} \cong 1$. This phenomenon partly explains why field saturation was not noted in previous simulation scans. Even with this masking effect, however, the accelerating peak still displays saturation when $\tilde{Q} \gg 1$, with increasing severity for $\tilde{Q}>100$. Examination of the useful acceleration amplitude, however, indicates that the accelerating field response is diminished above $\tilde{Q}=2$, just as is found for the average deceleration. For $\tilde{Q}>100$, the efficiency of exciting the acceleration field is very low. In fact, the useful acceleration diminishes more rapidly than the drive beam deceleration for large $\tilde{Q}$. This effect is more noticeable in the ultrashort beam simulation shown in Fig. 7, where the useful acceleration is smaller than the deceleration for even moderate $\tilde{Q}$.

There is, in the linear regime, a fixed relationship between acceleration and deceleration that is dependent only on beam geometry, not charge. The question then arises, where is the energy lost by the drive beam going, if not into acceleration? The answer is hinted at by the enhancement of this effect in the ultrashort, dense beam case - much of the energy that is deposited into the plasma by the beam does not, for plasma electrons initially close to the axis, go into generation of simple wave motion, but into very large amplitude scattered motion. While such electrons may seem, if one concentrates only on smaller radial regions, to be ejected in a near-ballistic manner from the beam region, in fact, they eventually lose energy, but at a different, much longer time scale than the main oscillation. These electrons, having a tight time profile, and a nontrivial density, may be considered to make up subsidiary "beams," which produce their own decelerating wakes. Since these wakes are far from the axis, and proceed with a long periodicity, such electrons do not contribute to building the initial accelerating wakefield, and their energy is effectively lost from the main component of wave system.

The generation of large radial-amplitude particles can be noted in the momentum-coded configuration space plot of Fig. 2, and the density/current profiles of Fig. 3. Other aspects of this phenomenon are shown in Fig. 9, which displays the longitudinal electric field associated with $\tilde{Q}=20$ cases, for both the long-beam geometry 

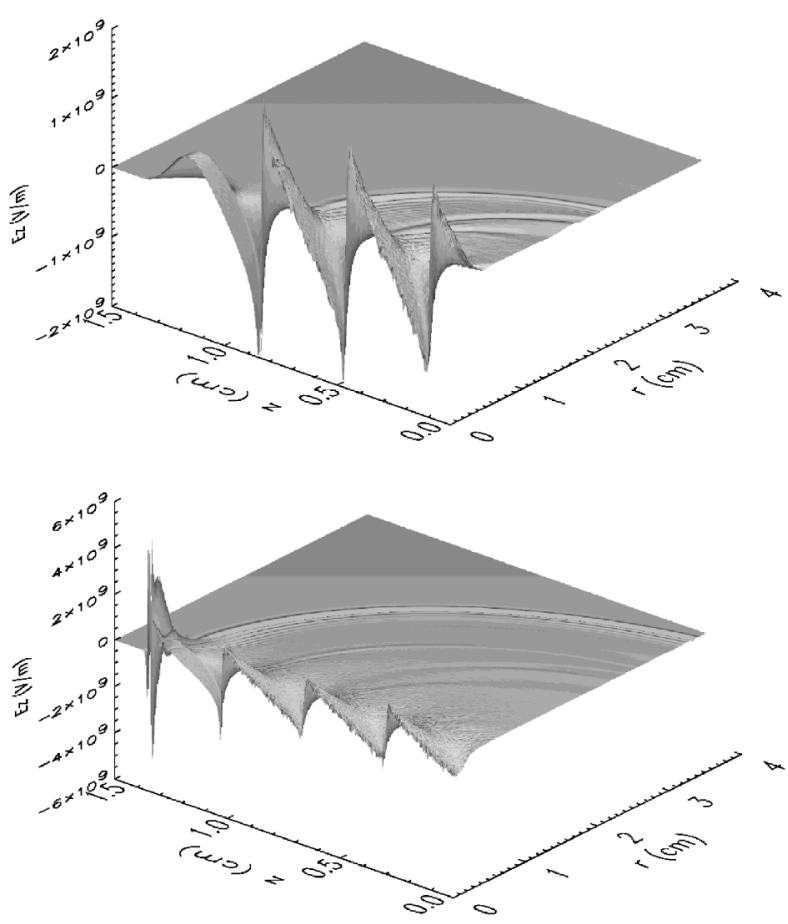

FIG. 9. Surface plot of $E_{z}$ in OOPIC PWFA simulation with $\tilde{Q}=20$, (a) long-beam geometry, as in Fig. 8, and (b) ultrashort beam geometry, as in Fig. 7.

[Fig. 9(a)] as in the simulations of Fig. 8 (similar to Fig. 2), and for the ultrashort beam [Fig. 9(b)] as in the simulations of Fig. 7. In the long-beam case, while there is negligible field disturbance electromagnetic energy density lost to large radial amplitudes emanating from the drive-beam region, there is a pronounced effect arising from the regions immediately following the field spikes that terminate the accelerating phases of the wake. These spikes are caused by electrons having very large momentum as they return to the axis, and this momentum is not removed during the dissolution of the spike. Thus many relativistic electrons are lost to subsequently large radial amplitudes. Associated with the wake of these electrons, which have a much shorter temporal length than the original drive beam, we again see significant levels of $E_{z}$.

The ejection of plasma electrons from the drive beam region to large radial amplitude is more severe for larger $\tilde{Q}$ cases. It is also a dominant effect in the ultrashort beam response, as seen in Fig. 9(b). A very large field response is observed propagating to large radial amplitudes in this case, one which clearly carries with it a significant fraction of the total electromagnetic energy deposited in the plasma (along with the mechanical energy associated with the motion). The severity of this effect for the ultrashort beam case illustrates the underlying mechanism behind the observation of accelerating field response that is lower than linear predictions, even for relatively small $\tilde{Q}$.

\section{EXPERIMENTAL SCALING WITH A GAUSSIAN BEAM-PLASMA SYSTEM}

The type of beam-plasma system scaling that has been explored in the simulations of Figs. 7 and 8 is what has been termed ideal. In performing these calculations, the plasma density and beam geometry were kept constant, while the charge was varied. This is of course exactly what one wants to do to conceptually understand the scaling of the system. It is not, however, what is strictly relevant to experiments. In accessing higher values of $\tilde{Q}$ in experiment, the beam is compressed longitudinally, ${ }^{3}$ and one typically then raises the plasma density to keep the condition $k_{p} \sigma_{z} \approx 1$. Thus $\tilde{Q}=4 \pi k_{p} r_{e} N_{b}$ is raised by increasing $k_{p}$ with $N_{b}$ constant. In ideal scaling, the beam density increases as $k_{p}^{3}$, while $n_{0}$ increases only as $k_{p}^{2}$, thus leading to higher values of $n_{b} / n_{0}$.

In order to accomplish ideal scaling [19], it is implied that the beam's matched beta function $\beta_{e q}$ scales as $k_{p}^{-1}$, which is in fact the case [3,4], as $\beta_{e q}=\sqrt{2 \gamma} k_{p}^{-1}$. On the other hand, it is clear that the beam emittance $\varepsilon$ does not decrease during compression; it can be at best constant, and may indeed increase due to collective effects [20,21] such as coherent synchrotron radiation. If one assumes that the beam emittance is held constant during compression, and that the beam is injected in a matched fashion to the ion focusing, then the normalized transverse beam size scales $\tilde{\sigma}_{r}=k_{p} \sqrt{\beta_{e q} \varepsilon} \propto \sqrt{k_{p}}$.

The results of a parametric study using what may be therefore termed "experimental" scaling are shown in Fig. 10. In these simulations, the normalized bunch length is held constant, $k_{p} \sigma_{z}=1.1$, while the normalized transverse (uniform density) beam size is given by $\tilde{a}=$ $0.2 \sqrt{\tilde{Q} / 2}$, as dictated by the constant emittance hypothesis. Note that because $\tilde{a}$ is now a function of $\tilde{Q}$, the extrapolated linear average deceleration and peak acceleration are no longer straight lines on the log-log plot. As the beam spot becomes larger with increasing $\tilde{Q}$, the electromagnetic coupling of the beam to the plasma decreases, as could have been anticipated by inspection of Eq. (3).

One interesting point in this regard is that the beam no longer becomes relatively denser than the plasma as $\tilde{Q}$ is increased, $n_{b} / n_{0} \propto \tilde{Q}^{0}$, and the relative density is stationary. In the example of Fig. 10, this ratio is 2.3, at the lower end of the blowout regime, and strong blowout is never accessed. As $\tilde{Q}$ is raised, however, the plasma response to the beam under experimental scaling also displays qualitatively different behavior - the plasma motion becomes increasingly longitudinal as $\tilde{a}$ approaches and exceeds unity, with the plasma return currents running inside the beam. When $\tilde{a} \gg 1$, one

\footnotetext{
${ }^{3}$ In many electron sources (e.g., rf photoinjectors with or without compressors) when one raises the charge, the bunch length also increases.
} 


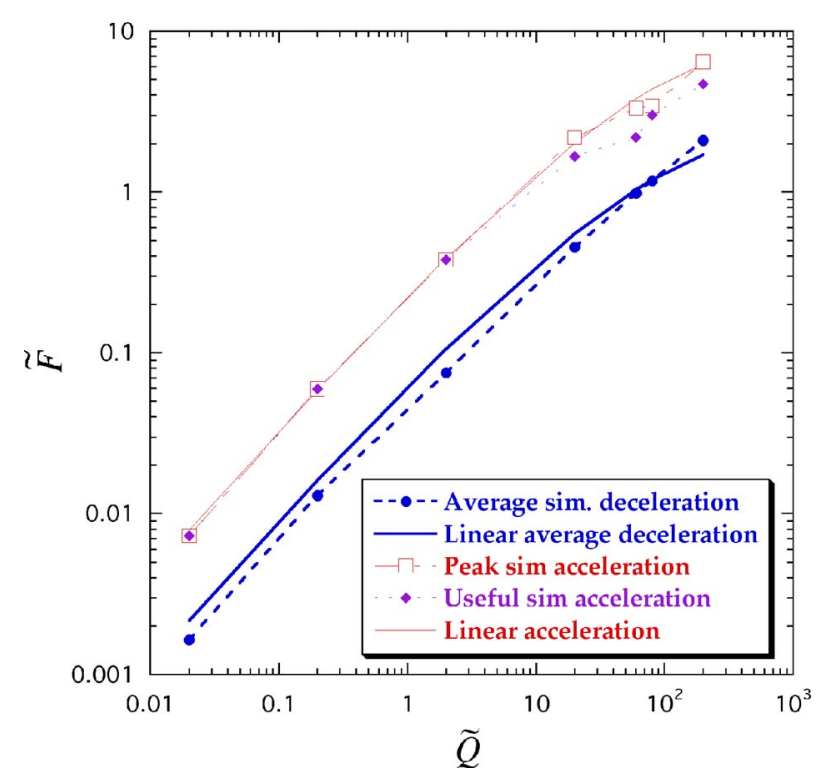

FIG. 10. (Color) The average normalized energy loss rate of $\tilde{F}_{\text {dec }}=e\left|\left\langle E_{z}\right\rangle\right| / m_{e} c \omega_{p}$ of a Gaussian-current electron beam with $k_{p} \sigma_{z}=0.1$, using experimental scaling, $\tilde{a}=0.2 \sqrt{\tilde{Q} / 2}$, as a function of $\tilde{Q}$, from linear theory (solid bold line) and selfconsistent PIC simulation (circles), the peak accelerating field behind the beam, $\tilde{F}_{\max }=e\left|E_{z, \max }\right| / m_{e} c \omega_{p}$, from linear theory (solid fine line) and PIC simulation (squares), and the useful field for acceleration (diamonds).

may expect that there is no relative reduction of beam coupling to the plasma with increasing charge, as beam does not eject the plasma electrons radially from its path. As such, the response in this limit does not resemble the blowout regime, but an inner diameter nonlinear system that was discussed previous to the proposal of the blowout regime [22].

These considerations shed light on the most striking aspect of the experimental scaling results of Fig. 10: the simulated deceleration and acceleration amplitudes are not notably different than those found from extrapolation of linear theory. Even the useful acceleration fields do not degrade from linear expectations by more than a factor of 2. The electric field response in this study is well approximated by the linear prediction, since the beam is not too much denser than the plasma, and the plasma response is not radial motion dominated as $\tilde{Q}$ increases to nominally "nonlinear" levels.

This study illustrates one of the expected laboratory pitfalls of experimental scaling - the plasma does not become more underdense when the beam is compressed, and eventually may note achieve blowout conditions. Thus one may expect the longitudinal fields to increase with compression, and according to linear expectations. But one may not, as indicated by simple arguments proposed by many previous authors, scale the expected field by $\sigma_{z}^{-2}$, without also noting the radial dependence implicit in the scaling of a constant emittance beam.
To illustrate this point, we take the case of the E164 experiment [15], now proposed for SLAC, as an extension to the E162 measurements [14]. In the first stage of E164, the beam will be compressed from $\sim 700$ to $\sim 100 \mu \mathrm{m}$, with the plasma density raised to keep $k_{p} \sigma_{z}$ approximately constant. This jump in density represents a scaling in $\tilde{Q}$ from roughly 1.5 to near 10 . An additional stage of E164 is more audacious, with the beam compressed to $\sim 12.5 \mu \mathrm{m}$, and a concomitant $\tilde{Q}$ of $\sim 80$. The parameters chosen for the radial beam size in our experimental scaling scan also correspond well to those achieved at the SLAC Final Focus Test Beam (FFTB) $\left(k_{p} \sigma_{r} \approx 2 \tilde{a} \approx\right.$ 0.2 at $\tilde{Q} \approx 2$ ), so this scan may be taken as a fairly accurate guideline for anticipating experimental scenarios. In fact, we have explicitly included the $\tilde{Q}=80$ point in order to compare the simulation to the approximate experimental conditions. Note that the scaling of $\tilde{Q}$ from 1.5 to 80 implies, using the linear extrapolation of field gradients (ideal scaling), that the acceleration should go from around $300 \mathrm{MeV} / \mathrm{m}$, as is predicted by the results in Fig. 10, and was experimentally observed [15], to nearly $850 \mathrm{GV} / \mathrm{m}$.

For the first stage of E164, experimental scaling the results of Fig. 10 gives a maximum acceleration gradient of $5 \mathrm{GV} / \mathrm{m}$, which is slightly larger than claimed in Ref. [15]. For the second stage of the E164 experiment, our scaling study predicts that the useful accelerating field is near $50 \mathrm{GV} / \mathrm{m}$. This is an impressive number, but is over an order of magnitude shy of the prediction given by the $\sigma_{z}^{-2}$ extrapolation of linear theory. It is also interesting to note in this context that if one could perform ideal scaling (by reducing the emittance along with bunch length), the nonlinearity of the plasma response, dominated by the radial ejection of the plasma electrons and concomitant loss of coupling, produces a useful acceleration which is nearly the same as in the experimental scaling case. Thus the nonlinear saturation of the plasma wave response with such a high value of $\tilde{Q}$ negates the advantage of using a narrower beam that is indicated by Eq. (3).

\section{CONCLUSIONS}

In conclusion, we have examined, through PIC simulation, aspects of the physics of the plasma electrons as they respond to a very high charge beam. We have concentrated on two areas of investigation: examination of the implications of analytical results concerning the fluid response derived in Ref. [2] and the scaling of the beamplasma interaction in both idealized and realistic experimental scenarios.

The simulations that most directly dealt with previous analytical predictions were performed with an ultrashort $\left(k_{p} l_{z}=0.1\right)$ beam. The most important qualitative analytical predictions, those of a strong initial forward component of the plasma electrons, along with the associated 
increase in density (and current density) excited by a large $\tilde{Q}$ beam, were examined in these simulations. In addition, the linearlike scaling of the deceleration fields driven by the beam was observed to occur up to extremely large $\tilde{Q}$, with significant deviations entering in only when $\tilde{Q}$ exceeded 20 . This is again is accordance with the most surprising of the analytical results given in Ref. [2]. This linearlike response in the fields stands in stark contrast to the nonlinear aspects (phenomena not associated with the linear theory) of the current density response that was observed even for $\tilde{Q}$ significantly smaller than unity. It is noted that the current density disturbance even for such a short beam leaves the immediate radial vicinity of the beam for $\tilde{Q} \gg 1$. This fast ejection of the plasma electrons results eventually in a loss of coupling between beam and plasma, and thus in lower than linear scaling of wakefield amplitude in $\tilde{Q}$.

In order to connect the nonlinear physics observed in analysis and theory for the ultrashort beam case to more experimentally relevant scenarios, a series of studies was undertaken with beam-plasma systems that have $k_{p} \sigma_{z}=$ 1.1. To explore the physics most characteristic of the PWFA blowout regime, an ideal scaling study, where both $k_{p} a$ and $k_{p} \sigma_{z}$ were held constant while $\tilde{Q}$ was varied, was performed. In this case, the decelerating fields inside the beam and the accelerating fields behind the beam displayed deviations from linear behavior. In particular, while the peak acceleration that is dominated by the spike at the tail of the accelerating portion of the wave scales nearly with linear expectations until $\tilde{Q}>20$, the useful field for acceleration is strongly degraded from the expectations of linear theory above $\tilde{Q}=2$. The masking of this degradation by reliance on measurement of the acceleration amplitude through the spike is noted as a probable cause for missing the deviations from linear scaling in previous studies.

The ideal scaling study definitively showed that while the previously proposed scaling of wakefield amplitudes as linear with $k_{p}^{2}$ (or $\sigma_{z}^{-2}$ for constant $k_{p} a$ and $k_{p} \sigma_{z}$ ) is remarkably persistent until $\tilde{Q}$ well exceeds unity for finite length beams, when $\tilde{Q}$ is large enough, the coupling of the beam to the plasma becomes much less efficient, and field amplitudes do not grow as expected. Further, for very high $\tilde{Q}$, plasma electrons that are strongly ejected in the radial direction, and therefore do not contribute to creation of a useful initial accelerating portion of the wake wave, form an increasingly large component of the energy lost by the drive beam to the plasma. For these reasons, it may be unwise to adopt this regime of the PWFA. We thus suggest that a practical limit of useful $\tilde{Q}$ for operation in the blowout regime is of the order of 10 .

On the other hand, for the presently proposed experiments that may access very large values of $\tilde{Q}$, these results may not apply. This is because when one compresses a beam of constant charge and emittance, the transverse beam size decreases only as $k_{p}^{-1 / 2}$, not as $k_{p}$. In such a case, while $\tilde{Q}$ increases due to the increase in $k_{p}$, the relative beam density does not. For proposed experiments at SLAC, this means that scaling to larger $\tilde{Q}$ through beam compression and use of a denser plasma does not imply moving into a more complete blowout scenario. In fact, since $k_{p} \sigma_{r}$ steadily increases under these conditions, for the most ambitious planned experiment $(\tilde{Q}=80)$, $k_{p} \sigma_{r}$ is near 1 , and the plasma response is more longitudinal than radial. In the experimental scaling study we have performed, where we take into account the growth in $k_{p} \sigma_{r}$ (which may be taken as approximately $\tilde{a} / \sqrt{2}$ for the sake of comparison between theory, simulation, and experiment) with increasing $\tilde{Q}$, it is found that all of these effects conspire to produce wakefield amplitudes that are very close to those predicted by linear theory, even for $\tilde{Q}=200$. It should be emphasized that this experimental scaling does not produce wakefields that are proportional to $k_{p}^{2}$ (or $\sigma_{z}^{-2}$ ), as previously proposed, because the beam-plasma coupling decreases with normalized beam radius $k_{p} \sigma_{r}$.

It should be noted that experimental scaling is dependent on the available beam emittance. As future highbrightness electron sources [23] should have emittances a factor of 10 better than those found currently at the SLAC FFTB, for the same parameters as the second phase of E164 proposes, $k_{p} \sigma_{r}$ would still be much less than 1 . In addition, the beam would be more than an order of magnitude denser than the plasma, and this experiment would be well into the blowout regime. In this $(\tilde{Q}=80)$ case, one may expect that the plasma wakefields would display significant degradation in amplitude due to nonlinear effects.

Finally, we end our discussion by noting that the plots of very nonlinear plasma disturbances shown in Figs. 3 and 9 emphasize that, while a notable amount of the energy deposited by the beam into the plasma is lost to creation of large amplitude particles that do not contribute to the acceleration phase of the wave, the majority of the plasma electrons taking part in the oscillation (especially in the more realistic long-beam case) engage in coherent motion. The degree to which these electrons stay localized together in both radial and longitudinal dimensions is remarkable. We may speculate that a more detailed examination of the microscopic physics of the plasma motion accompanying the formation of the accelerating phase of the wake may yet yield an explanation for such coherence.

\section{ACKNOWLEDGMENTS}

This work supported by U.S. Department of Energy Grant N. DE-FG03-92ER40693 and U.S. Department of Education Grant No. G1A-62056. 
[1] Pisin Chen, J.M. Dawson, Robert Huff, and T. Katsouleas, Phys. Rev. Lett. 54, 693 (1985); 55, 1537 (1985).

[2] N. Barov, J. B. Rosenzweig, M. C. Thompson, and R. Yoder, Phys. Rev. ST Accel. Beams 7, 061301 (2004).

[3] J. B. Rosenzweig, B. Breizman, T. Katsouleas, and J. J. Su, Phys. Rev. A 44, R6189 (1991).

[4] N. Barov and J. B. Rosenzweig, Phys. Rev. E 49, 4407 (1994).

[5] N. Barov, M. E. Conde, W. Gai, and J. B. Rosenzweig, Phys. Rev. Lett. 80, 81 (1998).

[6] C. Clayton et al., Phys. Rev. Lett. 88, 154801 (2002).

[7] S. Lee, T. Katsouleas, R. Hemker, and W. Mori, Phys. Rev. E 61, 7014 (2000).

[8] D. L. Bruhwiler et al., Phys. Rev. ST Accel. Beams 4, 101302 (2001).

[9] J. B. Rosenzweig, in Proceedings of the 1992 Linear Accelerator Conference, Ottawa, Ontario, Canada, 1992 (AECL-10728, 1993).

[10] J. B. Rosenzweig et al., Nucl. Instrum. Methods Phys. Res., Sect. A 410, 532 (1998).

[11] N. Barov et al., Phys. Rev. ST Accel. Beams 3, 011301 (2000).

[12] J. D. Jackson, Classical Electrodynamics (Wiley, New York, 1975), 2nd ed.
[13] N. Barov et al., in Proceedings of the 2001 Particle Accelerator Conference, Chicago, 2001, edited by P. Lucas and S. Webber (IEEE, New York, 2002), p. 126.

[14] M. Hogan et al., Phys. Plasmas 7, 2241 (2000).

[15] P. Muggli et al., in The Physics and Applications of High Brightness Electron Beams, edited by J. B. Rosenzweig, L. Serafini, and G. Travish (World Scientific, Singapore, 2003).

[16] S. Lee et al., Phys. Rev. ST Accel. Beams 4, 011001 (2002).

[17] R. D. Ruth and A.W. Chao, in Laser Acceleration of Particles, edited by P. Channell, AIP Conf. Proc. No. 91 (AIP, New York, 1982).

[18] D. Smithe et al., Comput. Phys. Commun. 78, 54 (1995).

[19] J. B. Rosenzweig and E. Colby, in Advanced Accelerator Concepts, edited by P. Schoessow, AIP Conf. Proc. No. 335 (AIP, New York, 1995), p. 724.

[20] H. Braun, Phys. Rev. Lett. 84, 658 (2000).

[21] S. Heifets, G. Stupakov, and S. Krinsky, Phys. Rev. ST Accel. Beams 5, 064401 (2002).

[22] J. B. Rosenzweig, Phys. Rev. Lett. 58, 555 (1987).

[23] M. Ferrario et al., in Proceedings of the European Particle Accelerator Conference, Vienna, 2000 (European Physical Society, Vienna, 2000), p. 1642. 